\title{
A Rare Case of Imatinib-Induced Pneumonitis
}

\author{
*Geoffrey Watson, Sarah Picardo, Philip Hodnett, Julie O’Brien and Nemer Osman \\ Department of Medical Oncology and Radiology, University Hospital of Limerick, Ireland
}

Submission: September 13, 2016; Published: September 22, 2016

*Corresponding author: Geoffrey Watson, Department of Medical Oncology, University Hospital Limerick, St. Nessan's Road, Dooradoyle, Co. Limerick, Ireland, Tel: +961-1-350-000; Fax: +9611370795; Email: Geoffrey.watson@hse.ie

\begin{abstract}
Gastrointestinal stromal tumors (GISTs) are rare mesenchymal neoplasms that may affect any portion of the gastrointestinal tract. Surgical resection was previously considered the only treatment modality available, thus prognosis in patients with advanced disease was poor. The emergence of novel therapeutic agents such as imatinib mesylate has altered how physicians now approach and manage these unique malignancies. The addition of these targeted agents has resulted in a significant benefit in median overall survival. Imatinib treatment is generally well tolerated. Side effects such as oedema, rash and gastrointestinal upset may be observed. However respiratory complaints are exceedingly rare. We report a case of imatinib-induced interstitial pneumonitis after five months of treatment in a patient with intermediate risk gastrointestinal stromal tumor of the stomach.
\end{abstract}

Keywords: Imatinib Mesylate; Glivec; Interstitial lung disease; Pneumonitis; Gastrointestinal stromal tumor (GIST)

Abbreviations: GISTs: Gastrointestinal Stromal Tumors; CML: Chronic Myeloid Leukemia; OGD: Oesophagogastrodudoenoscopy; ATP: Adenosine Triphosphate; TKI: Tyrosine Kinase Inhibitor; TAP: Thorax, Abdomen \& Pelvis; PFS: Progression Free Survival; OS: Overall Survival; HRCT: High Resolution CT; PDGF: Platelet-Derived Growth Factor.

\section{Introduction}

Gastrointestinal stromal tumors (GISTs) are rare malignancies. GISTs belong to the family of mesenchymal tumors, which represent only $1 \%$ of all primary GI cancers $[1,2]$. Historically surgery was the only treatment available for GISTs, thus prognosis in those with advanced disease was poor. Advances in our understanding of these tumors at a molecular level, namely the pivotal discovery that the mutational activation of KIT genes stimulates the growth of these cancer cells, have revolutionized treatment. This breakthrough paved the way for the development of novel therapeutic agents such as imatinib mesylate (Glivec, Novartis) which has been shown to increase median survival to 60 months [2,3]. This tyrosine kinase inhibitor also targets the BCR-ABL fusion oncoprotein, rendering it as a useful therapeutic agent in chronic myeloid leukemia (CML).

Imatinib treatment is generally well tolerated. Discontinuation due to intolerance is uncommon, affecting approximately $5 \%$ of patients [4]. The most frequent side effects observed during therapy include peripheral oedema, pleural or pericardial effusion, ascites, nausea and vomiting, myalgia, diarrhea and skin rash $[4,6]$. Respiratory side effects of imatinib are relatively rare, with an incidence of $0.2-1.3 \%$ [7].

We report a rare case of imatinib-induced interstitial pneumonitis after five months of treatment in a patient with intermediate risk gastrointestinal stromal tumor of the stomach.

\section{Case Report}

A 65 year old female presented with a three week history of vague abdominal discomfort and intermittent vomiting. She denied any history of fevers, altered bowel habits or weight loss. Her background was significant for a right oophorectomy 33 years previously and left salpingo-oophorectomy 24 years ago, both for benign conditions. She took no regular medications and reported no drug allergies. She had no significant family history of cancer. She was an ex smoker having quit over 30 years ago with a 10 pack year history. She did not consume alcohol.

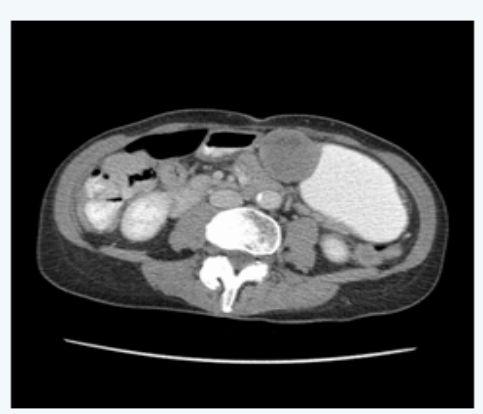

Figure 1: CT Abdomen demonstrating a large gastrointestinal tumour of the stomach.

Physical examination revealed mild epigastric discomfort on palpation. Routine blood panel was unremarkable. 


\section{Cancer Therapy \& Oncology International Journal}

Oesophagogastrodudoenoscopy (OGD) and colonoscopy were normal. CT imaging of her thorax, abdomen and pelvis (TAP) revealed a $4.5 \mathrm{~cm}$ solid lesion of the lesser curvature of the stomach, suspicious for GIST (Figure 1). There was no evidence of metastatic disease elsewhere. She was discussed at the gastrointestinal multidisciplinary meeting and preceded to laparoscopic partial gastrectomy. Pathology revealed an intermediate grade GIST. The tumor was $5 \mathrm{~cm}$ in size with eight mitoses per 50 high-powered fields. Risk stratification as per Miettenen and Lasota (2006) placed her in the intermediate risk group with a $16 \%$ chance of progressive disease. A supplementary pathology report revealed an exon 11 activating mutation. Baseline imaging post surgery revealed no evidence of metastatic disease. She was re-discussed at the sarcoma multidisciplinary meeting and the decision was made to commence adjuvant imatinib therapy at a dose of $400 \mathrm{mg}$ once daily.
Five months later she attended our surveillance outpatient clinic for review. Clinically she reported feeling well, however after further questioning she revealed she did suffer from mild shortness of breath on exertion. She denied any cough or chest pain. A re-staging CT TAP revealed no evidence of disease recurrence; however interval onset of multisegmental, sub solid and pure ground-glass pulmonary nodules was reported, with appearances favoring imatinib-induced pneumonitis (Figure 2). Routine blood panel including inflammatory markers were again unremarkable. Eosinophils were normal (0.17 x 109 /L). Imatinib was discontinued and she was started on a short course of steroids. Interval follow up high resolution CT (HRCT) at eight weeks revealed marked improvement in the ground glass changes previously observed (Figure 3). She returned to clinic and reported no respiratory symptoms. She was Re-challenged with Imatinib at the same dose of $400 \mathrm{mg}$ once daily. She will remain under close surveillance.

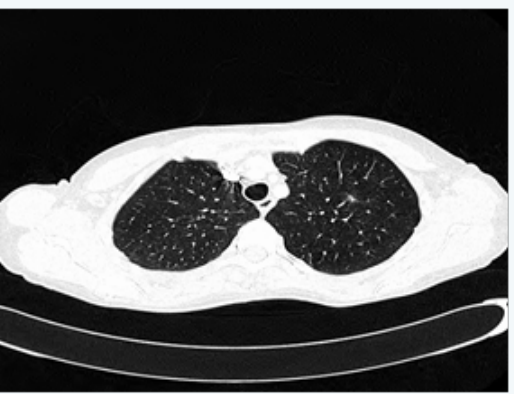

(A)

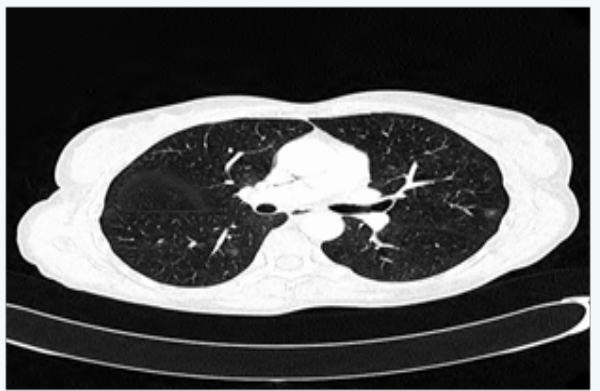

(B)

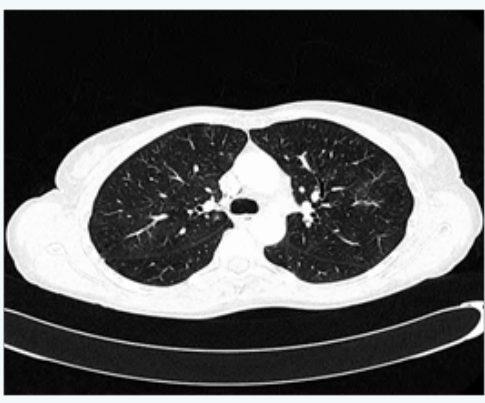

(C)

Figure 2: (A-C) CT Thorax demonstrating interval onset of ill-defined part solid $(A)$ and pure ground glass $(B, C)$ pulmonary nodules suggestive of Imatinib-induced pneumonitis.

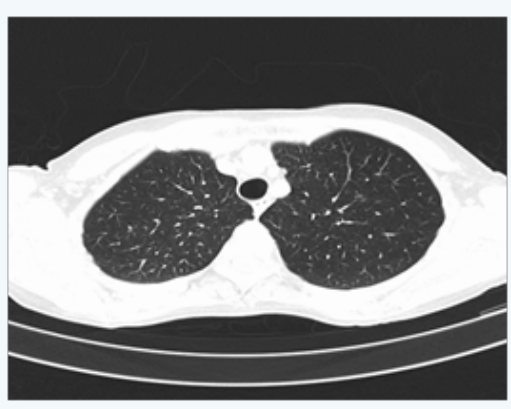

(A)

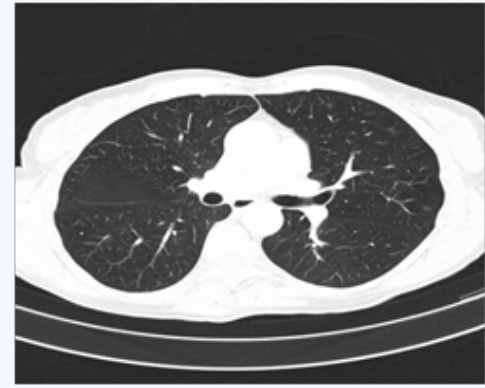

(B)

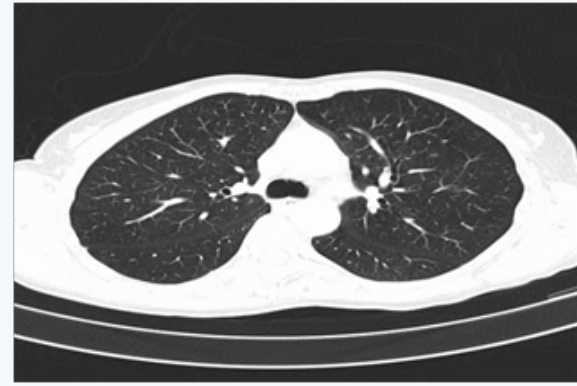

(C)

Figure 3: (A-C) High Resolution CT (HRCT) Thorax demonstrating decreased multisegmental ground glass attenuation consistent with resolving pneumonitis.

\section{Discussion}

Mesenchymal tumors are the most common benign nonepithelial neoplasms affecting the GI tract [1,4]. GISTs represent a subset of this family and may be found in any portion of the GI tract but are most commonly localized to the stomach and proximal portions of the small intestine. Median age of diagnosis is 66-69 years [4].

In 1998 Hirota et al. discovered that GISTs arise from gainof-function mutations in c-KIT, a transmembrane receptor protein kinase with a role in regulating cellular proliferation, differentiation, adhesion and apoptosis [8]. This pivotal breakthrough paved the way for the development of novel therapeutic agents such as Imatinib mesylate.

Imatinib mesylate (Glivec) is a tyrosine kinase inhibitor (TKI) that structurally mimics adenosine triphosphate (ATP). It binds competitively to the ATP binding site of the target kinase, preventing substrate phosphorylation and signaling, thereby inhibiting tumor proliferation [9]. 
The addition of imatinib in the adjuvant setting has been shown to improve progression free survival (PFS) and Overall Survival (OS) in postsurgical patients, with at least 36 months of treatment in patients with an intermediate or high risk of recurrence [10].

In general imatinib is well tolerated. The most common side effects reported in the literature include fluid retention, diarrhea, nausea, fatigue, muscle cramps, abdominal pain and rash; however these effects are generally grade 1-2 and tend to improve with prolonged therapy. Congestive heart failure has been noted in $8.2 \%$ of patients, while arrhythmias and acute coronary syndromes have also rarely been reported [4]. All toxicities subside with discontinuation of treatment.

Respiratory side effects are uncommon, however cough, dyspnoea and upper respiratory tract infections have been reported [6]. Dyspnoea in particular may be due to pulmonary oedema. It has been postulated that the fluid retention associated with imatinib therapy may be due to prolonged platelet-derived growth factor (PDGF) inhibition, which plays a role in the regulation of interstitial fluid homeostasis [4,7].

The development of pulmonary fibrosis and pneumonitis as a side effect of imatinib treatment is exceedingly rare and has only been described in isolated case reports to date [4]. The largest case review by Ohnishi et al. [7] included 5500 patients on Imatinib therapy; with only 27 cases of interstitial lung disease reported.

Imatinib pneumonitis appears to develop on average approximately two months after commencing treatment with a median dose of $400 \mathrm{mg}$ imatinib [4,5]. Incidence appears to be higher in those with pre-existing lung disease [5]. In the present case, the development of pneumonitis occurred after five months of treatment.

Diagnosis may be challenging as signs and symptoms are often non-specific. Malaise, low-grade fever, dyspnea and cough appear to predominate [11]. Lab tests may reveal eosinophilia, although in our patient a routine blood panel was unremarkable.

Radiologic investigations often narrow the differential diagnosis. Chest radiographs may reveal interstitial pulmonary infiltrates. CT thorax may reveal diffuse or patchy groundglass opacity, consolidation, or fine nodular opacity. Six different radiologic patterns have previously been described as suggestive of drug-related interstitial lung disease $[7,11,12]$. The hypersensitivity reaction pattern is described as diffuse homogenous or widespread opacity, represented by groundglass attenuation. This pattern lacks traction bronchiectasis and structural distortion. The interstitial pneumonia pattern displays ground-glass attenuation and irregular linear or reticular areas of attenuation with associated bronchiectasis and bronchiolectasis. The cryptogenic-organizing pneumonia (pattern is associated with non-segmental abnormal opacities resembling consolidation in a sub pleural or peribronchovascular distribution. The nodular pattern presents as diffuse fine nodular opacities along the bronchovascular bundle. The peribronchovascular bundle pattern, which can be described as patchy ground glass changes predominantly affecting the bronchovascular bundle. The final radiologic pattern is termed the diffuse alveolar damage pattern [7]. Our patient's imaging was suggestive of the hypersensitivity type of reaction.

Pathological examination may show interstitial pneumonitis and fibrosis, destruction of alveolar septa, lymphocytic alveolitis, plasma cell infiltrates, or type II pneumocyte hyperplasia.

The mechanism of interstitial lung diseases remains speculative. It is thought to be the result of an immune complexmediated reaction involving $\mathrm{T}$ cells and cytokines. If allowed to progress, the inflammation can extend to interstitium and its capillaries, leading to pneumosclerosis, alveolar deformation and disturbances of lung diffusion capacity [4]. In addition, it has been postulated that imatinib may aggravate interstitial pneumonitis by inhibiting PDGF tyrosine kinase, which leads to acute lung injury $[13,14]$.

Management of drug-induced pneumonitis involves immediate discontinuation of the offending agent. Administration of corticosteroids is optional depending on the severity of the patient complaints and clinical signs. Serial imaging may be required to ensure resolution. A re-challenge with imatinib may be considered when the patient has displayed improvement both clinically and radiologically $[5,15]$.

\section{Conclusion}

Drug-induced pneumonitis is a rare complication of imatinib treatment. Initial diagnosis may be challenging due to nonspecific signs and symptoms, and may be mistaken for more common infectious differentials. Thus awareness and clinical suspicion is paramount for early detection and appropriate management.

\section{References}

1. Rubin BP, Fletcher JA, Fletcher CD (2000) Molecular Insights into the Histogenesis and Pathogenesis of Gastrointestinal Stromal Tumors. Int J Surg Pathol 8(1): 5-10.

2. Watson GA, Kelly D, Melland-Smith M, Gleeson J, McEntee G, et al. (2016) Get the GIST? An Overview of Gastrointestinal Stromal Tumours. Ir J Med Sci 185(2): 319-326.

3. Otani Y, Furukawa T, Yoshida M, Saikawa Y, Wada N, et al. (2006) Operative indications for relatively small $(2-5 \mathrm{~cm})$ gastrointestinal stromal tumor of the stomach based on analysis of 60 operated cases. Surgery 139(4): 484-492.

4. Lazareva OV, Turkina AG (2012) Drug- Induced Pneumonitis: A Rare Complication of Imatinib Mesylate Therapy in Patients with Chronic Myeloid Leukemia, Dr Steffen Koschmieder (Edn.), InTech.

5. Go SW, Kim BK, Lee SH, Lee JM, Hah JH, et al. (2013) Successful Rechallenge with Imatinib in a Patient with Chronic Myeloid Leukemia Who Previously Experienced Imatinib Mesylate Induced Pneumonitis. Tuberc Respir Dis 75(6): 256-259. 


\section{Cancer Therapy \& Oncology International Journal}

6. Turkina AG, Khoroshko ND, Druzhkova GA, Zingerman BV, Zakharova ES, et al. (2005) Recommendations for the treatment of patients with chronic myeloid leukemia.

7. Ohnishi K, Sakai F, Kudoh S, Ohno R (2006) Twenty-seven cases of drug-induced interstitial lung disease associated with imatinib mesylate. Leukemia 20(6): 1162-1164.

8. Hirota S, Isozaki K, Moriyama Y, Hashimoto K, Nishida T, et al. (1998) Gain-of-function mutations of c-kit in human gastrointestinal stromal tumors. Science 279(5350): 577-80.

9. Sepe PS, Brugge WR (2009) A guide for the diagnosis and management of gastrointestinal stromal cell tumors. Nat Rev Gastroenterol Hepato 6(6): 363-371.

10. Joensuu H, Eriksson M, Sundby Hall K, Hartmann JT, Pink D, Schütte J, et al. (2012) One vs three years of adjuvant imatinib for operable gastrointestinal stromal tumor: a randomized trial. JAMA 307(12): 1265-1272.
11. Peerzada MM, Spiro TP, Daw HA (2011) Pulmonary toxicities of tyrosine kinase inhibitors. Clin Adv Hematol Oncol 9(11): 824-836.

12. Rossi SE, Erasmus JJ, McAdams HP, Sporn TA, Goodman PC (2000) Pulmonary drug toxicity: radiologic and pathologic manifestations. Radiographics 20(5): 1245-1259.

13. Yokoyama T, Miyazawa K, Kurakawa E, Nagate A, Shimamoto T, et al. (2004) Interstitial pneumonia induced by imatinib mesylate: pathologic study demonstrates alveolar destruction and fibrosis with eosinophilic infiltration. Leukemia 18(3): 645-646.

14. Snyder LS, Hertz MI, Peterson MS, Harmon KR, Marinelli WA, et al (1991) Acute lung injury: pathogenesis of intraalveolar fibrosis. J Clin Invest 88(2): 663-673.

15. Delomas T, Darne C, Besson C (2012) Lack of recurrence of imatinibinduced interstitial lung disease with nilotinib. Leuk Lymphoma 53(2): 332-333. 